\title{
Implementation of Internal Mixed Signal ESD Protection onto RFID Transponder IC
}

\author{
M. K. Khaw ${ }^{1}$, F. Mohd-Yasin ${ }^{1}$,Member, IEEE, Y. K. Teh ${ }^{1}$, Student Member, IEEE, M B.I. Reaz ${ }^{2}$, \\ Member, IEEE \\ Faculty of Engineering, Multimedia University, 63100 Cyberjaya, Selangor, MALAYSIA \\ ${ }^{2}$ ECE Dept, IIUM, 53100 Kuala Lumpur, MALAYSIA \\ Phone: $+603-83125423$ \\ Fax: $+603-83183029$ \\ Email: khaw.mei.kum@mmu.edu.my
}

\begin{abstract}
Radio Frequency Identification (RFID) has become an important wireless data communication tool in recent years. As much as we want to ensure data integrity and the robustness of the RFID transponder, Electrostatic Discharge (ESD) influence on the transponder can jeopardize it. Current practice put the ESD protection in the package. However at pad level, the ESD protection is usually small dimensioned to reduce input capacitance. Hence extra ESD protection co-constructed at internal circuit VDD-VSS rail is necessary in advanced process due to thinner gate oxide. In this paper, we have developed an internal ESD protection circuit and implemented it in our previously developed $13.56 \mathrm{MHz}$ RFID transponder employing TSMC $0.18 \mu \mathrm{m}$ process. The circuit has a capability to sustain 2-KV of HBM positive mode ESD voltage, which is suitable for RFID applications. The additional power consumption for the clamp circuit is only $15.12 \mathrm{nW}$.
\end{abstract}

\section{INTRODUCTION}

Radio Frequency Identification (RFID) is an important data communication tool due to its contactless technology and the ability of its transponder to be rewritable, having large memory capacity and able to withstand visually and environmentally challenged conditions. Just like many electronic devices, the RFID transponder is no less vulnerable from the Electrostatic Discharge (ESD) damages. ESD is a transfer of energy between two materials of different electrostatic potential. For example, human can transfer the ESD to the chip he/she is holding from touching a chair. While ESD can be potentially eliminated during manufacturing process by taking stringent measures in the whole production of the tag [1-4], it is not easily avoidable in real life application. As the RFID transponders are progressively used in logistics and retails, it is exposed to a lot of human touches. Therefore, the ESD protection of these transponders is vital.

Nowadays, many manufacturers put the ESD protection in the package. However at pad level, the ESD protection is usually small dimensioned to reduce input capacitance. This is why extra ESD protection co-constructed at internal circuit VDD-VSS rail is necessary in advanced process due to thinner gate oxide.

There were some discussions and works [5-9] done to improve power rail ESD robustness in the mixed-signal circuits. Most ESD protection is usually accomplished by using various types of clamp devices to shunt the electrical charge away from internal circuits before over voltage damage can occur. The clamp designs can consist of diodes and/or MOSFETs. Works by Ming-Dou Ker, Kei-Kang Hung et al. [5] shown in Figure 1 and 2, use the power-rail ESD clamp circuit with stacked gate-grounded diodes and also the gatetriggered diodes. The gate-triggered design proved to have a higher ESD robustness and faster turn-on speed compared to the gategrounded design as shown in Figure 3, due to the existence of the RC-based detection circuit. 


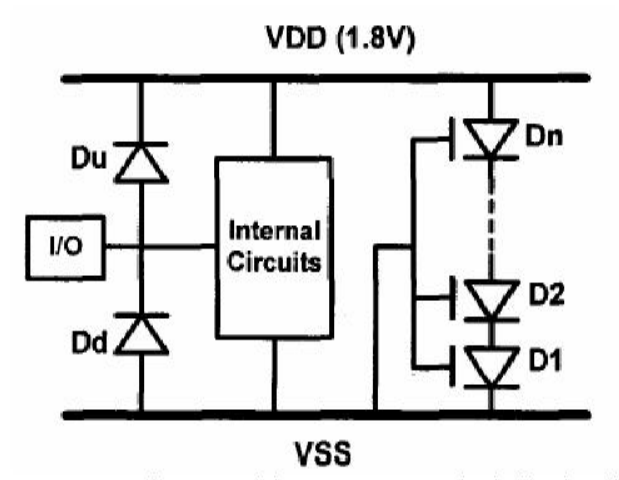

Figure 1 ESD clamp with gate-grounded diodes [5]

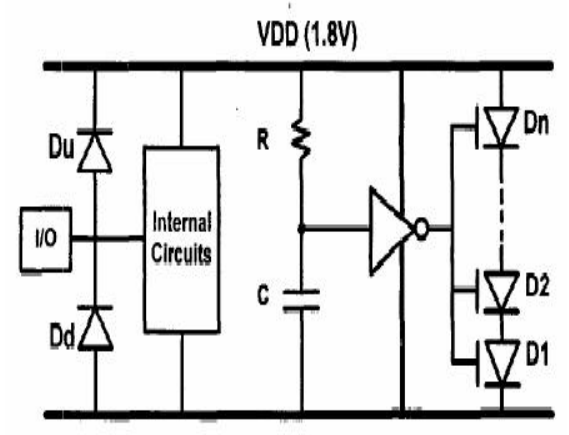

VSS

Figure 2 ESD clamp with gate-triggered diodes [5]

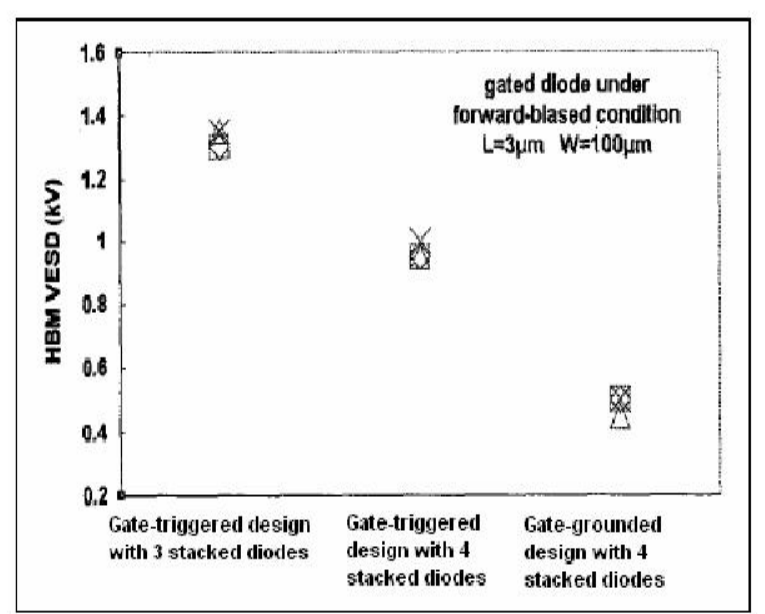

Figure 3 Comparison results of the gate-grounded and gate-triggered diodes [5]

Improvement by Ming-Dou Ker and CheHao Chuang [6] has a PMOS (NMOS) inserted into the diode structure to form the PMOSbounded (NMOS-bounded) diode as shown in Figure 4 and 5, which is essential to block the field oxide isolation across the $\mathrm{p} / \mathrm{n}$ junction in the diode structure. Thus, the PMOS/NMOSbounded diodes can sustain much higher ESD stress, especially under the reverse-biased condition.
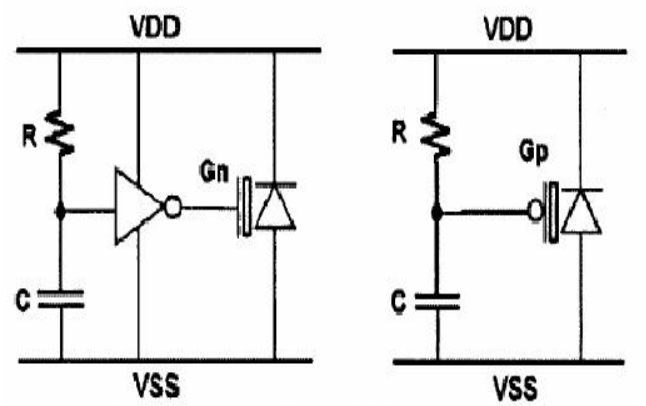

Figure 4 ESD clamp with RC-based detection circuit to control the NMOS and PMOS-bounded diode [6]
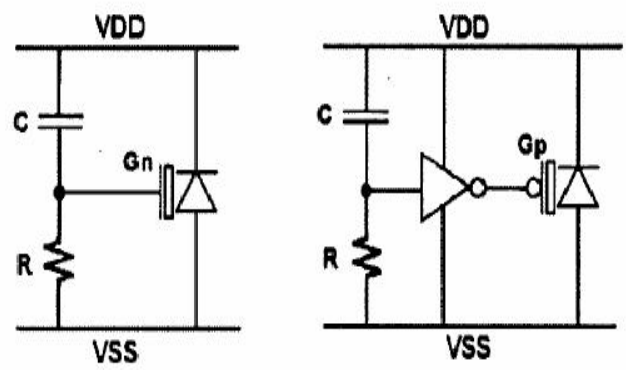

Figure 5 ESD clamp with gate-coupled technique to control NMOS and PMOS-bounded diode [6]

From the tests shown in Figure 6, the PMOS (NMOS)-bounded diodes have much higher ESD tolerance than the normal diodes and polybounded diodes. However, ESD clamps using gate-grounded NMOS has higher tolerance to ESD levels than the MOS bounded diodes. The only drawback of the gate-grounded NMOS would be the non-uniformity turned-on due to multiple fingers that could occur.

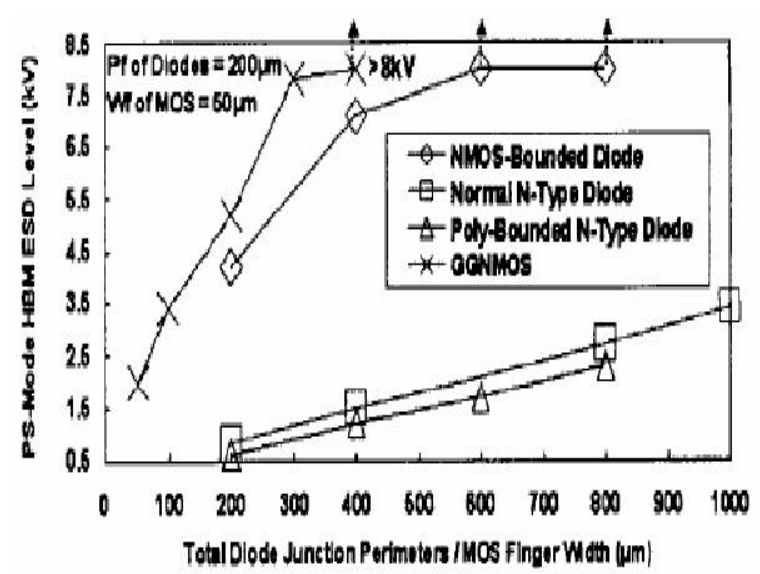

Figure 6 Comparison of various ESD clamps [6]

Other kinds of ESD clamps using MOSFETs are the NMOS gate-driven clamp design [7-8] and substrate-triggered clamp design [9]. The latter design is developed by Ming-Dou Ker et al. which consists of a substrate-triggering field oxide device (STFOD) as a replacement for the 
NMOS. Figure 7 shows the device structure of STFOD.

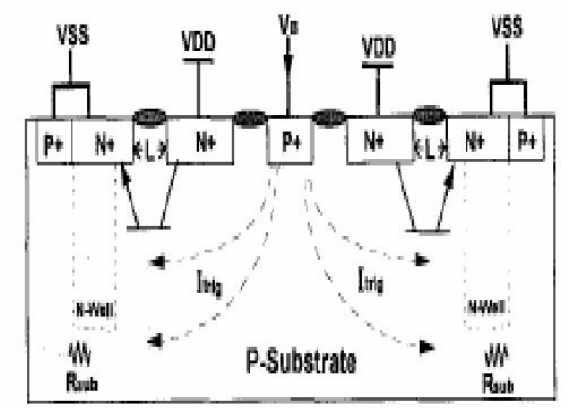

Figure 7 Substrate-triggered clamp design (STFOD) [9]

It is reported to perform better, in terms of layout area and efficiency, compared to the NMOS clamp. However, none were implemented for the RFID IC Transponder. In our work, we use gate-driven technique employing NMOS as it is simpler and very suitable to work under a 2KV Human Body Model (HBM) positive mode ESD voltage. The clamp is implemented throughout a $13.56 \mathrm{MHz}$ RFID transponder. The RFID transponder had been developed in this project previously. The 2-KV Human Body Model (HBM) positive mode ESD voltage is used as a reference point to test the effectiveness of the protection circuit. 2-KV HBM is the commercial IC product ESD level.

The following section introduces the architecture of the mentioned ESD protection circuit and its implementation in the RFID transponder. Section III shows the results when we applied a 2-KV Human Body Model (HBM) positive mode ESD voltage pulse to the ESD protected circuit and some discussions. Finally, section IV shows the conclusion.

\section{ARCHITECTURE}

The idea of the ESD protection circuit is to clamp existing ESD voltage on the VDD power line in the circuitry to the ground so that ESD pulses will not enter the main circuitry and damage it. Figure 8 shows the circuit of the VDD-to-VSS ESD clamp using the NMOS gatedriven technique. The clamp consists of a transient detection circuit which is the resistor (R), source-drain grounded NMOS acting as a capacitor (C), an NMOS (M3) and a PMOS
(M2); and an NMOS (M1) to bypass the ESD voltage to the ground.

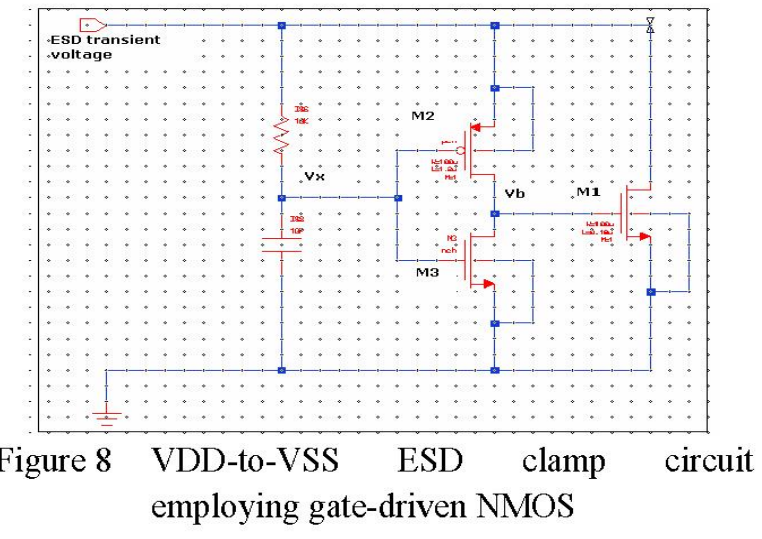

The clamp is designed to be turn on only under the ESD stress conditions but kept off under normal operating conditions. The resistor and capacitor are set to $0.1 \mu \mathrm{s}$ to differentiate and detect the transient ESD voltage from the useful VDD. Initially, the nodes $\mathrm{Vx}$ and $\mathrm{Vb}$ are $0 \mathrm{~V}$. The ESD voltage across the VDD power line will then charge the capacitor to rise up to the voltage of $V x$. Since the HBM ESD voltage with 2-KV charge has a rise time of about 5-10ns, the voltage level of $\mathrm{Vx}$ is increased much slower than the voltage level on VDD line. Due to the delay in voltage increase on Vx, M2 is biased by the ESD voltage and sends a voltage into node $\mathrm{Vb}$ to turn on M1. As long as the voltage on the node $\mathrm{Vb}$ is greater than the threshold voltage of the transistor, M1 is turned on and it will provide a short circuit path between the VDD and the ground. Under normal operating condition, VDD has a rise time in the order of $3 \mu \mathrm{s}$. With such rise time, the ESD clamp with RC of $0.1 \mu \mathrm{s}$ will be able to keep $\mathrm{Vb}$ with $0 \mathrm{~V}$ voltage level. Therefore, Ml is kept off throughout the normal operating condition. 


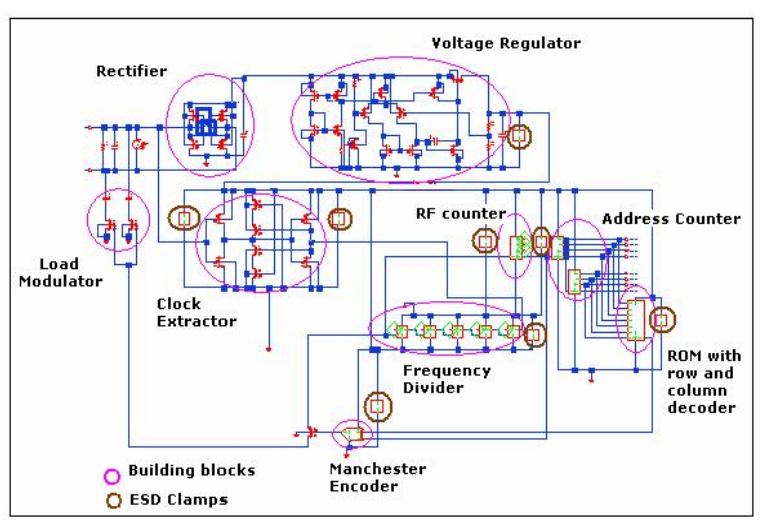

Figure 9 Implementation of ESD clamps on the transponder IC

Figure 9 shows the implementation of the clamps to the entire RFID transponder architecture. An ESD clamp is assigned to each building block that is connected to VDD. It is not necessary to include the ESD protection circuit in the RF front end as they will be taken care off by the standard input and output pad ESD protection since in our work; we only concentrate on the VDD-VSS ESD protection.

There are in total 8 ESD clamps. The ESD clamps are inserted in very near to each building block because VDD is the main voltage source and it is generally spread out in the entire transponder IC. This is to ensure the efficiency of the ESD protection circuit as longer power lines can cause a time delay to discharge ESD voltage through the ESD clamps [7].

\section{RESULTS AND DISCUSSION}

We use the Mentor Graphic Design Architect for the schematic entry and Eldo as the simulator. The transponder and proposed ESD clamps are all done according to TSMC $0.18 \mu \mathrm{m}$ Mixed Signal SALICIDE (1P6M, $1.8 \mathrm{~V})$ process. After implementing the ESD clamps into the transponder, we simulate the circuit with two conditions. First, we add a 2 $\mathrm{kV}, 400 \mathrm{~ns}$ pulse with a rise time of $10 \mathrm{~ns}$ to the VDD. This stimulus acts as the ESD pulse that could occur on the power line. Figure 10 shows the circuit performance. We can see that $\mathrm{Vb}$ starts to increase slowly with its voltages above the threshold voltage when the $2 \mathrm{kV}$ is fed to the ESD clamp. At this time, we observed that M1 is turned on. This shows that the VDD-to-VSS ESD clamp circuit had successfully diverted the large current to ground, and avoid damage to the circuit.

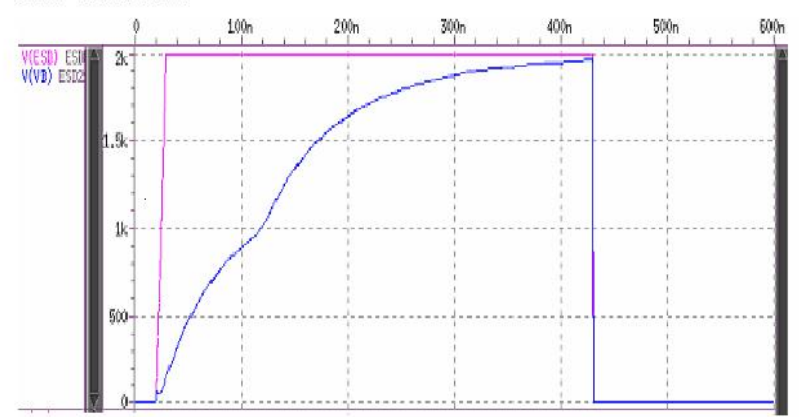

Figure $10 \mathrm{ESD}$ transient pulse at $2000 \mathrm{~V}$ with the corresponding $\mathrm{Vb}$

Figure 11 shows the circuit performance under the normal condition. As the stimulus, we add a pattern with a $20 \mu$ s fall time to represent VDD without the influence of the ESD pulse. Vb is kept almost zero. The ripple occurred shows that it is below the threshold voltage of the transistor, therefore M1 is kept off the whole time. No current is bypassed to the ground.

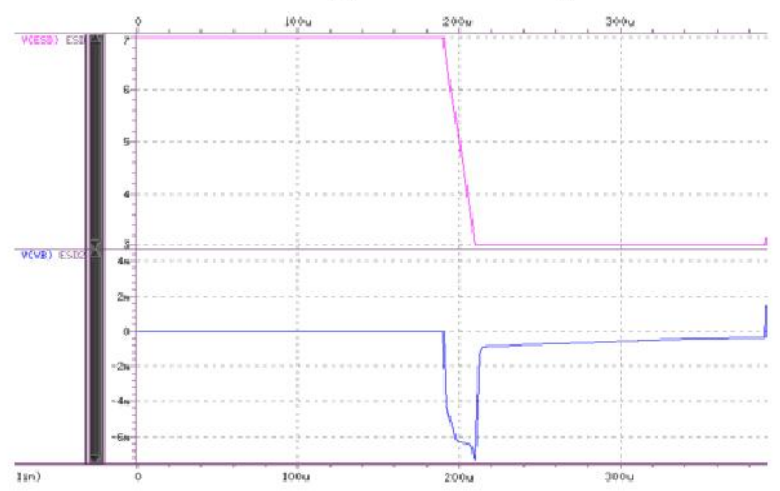

Figure 11 ESD transient pulse at $7 \mathrm{~V}$ during normal operation

The power consumption of the transponder circuit with the clamps is $1.2344405 \mathrm{~mW}$ while the power consumption of the transponder circuit without the clamps is $1.2344253 \mathrm{~mW}$. The difference is about $15.12 \mathrm{nW}$.

\section{CONCLUSION}

We have developed an internal ESD protection circuit and implemented it in the previously developed $13.56 \mathrm{MHz}$ RFID transponder employing TSMC $0.18 \mathrm{um}$ process. The circuit has a capability to sustain $2-\mathrm{KV}$ of HBM positive mode ESD voltage, which is suitable for RFID applications. The power consumption with the clamp circuits is only an 
addition of $15.12 \mathrm{nW}$, which shows successful power efficient design.

\section{ACKNOWLEDGEMENT}

The authors would like to thank the Malaysian Ministry of Science, Technology and Environment for sponsoring this work under project IRPA 03-99-01-0093 EA091.

\section{REFERENCES}

[1] Mark Blitshteyn, "Mastering RFID Label Converting". White Paper, RFID Journal. Published July 2006.

[2] Basics of Electrostatic Discharge, ESD Association, www.esda.org, 2000.

[3] Mykkanen, C. Fred and Blinde, David R., The Room Air Ionization System, a Better Alternative than $40 \%$ Relative Humidity, Electrical Overstress/Electrostatic Discharge Symposium Proceedings,Las Vegas, 1983.

[4] Shaw, Monica, Pushing Past Paper, Pulp \& Paper, November 2004.

[5] Ming-Dou Ker, Kei-Kang Hung, Howard T.-H. Tang, S.-C. Huang, S.-S. Chen and M.-C. Wang, "Novel Diode Structures and ESD Protection Circuits in a 1.8 -V 0.15-pm Partially-Depleted SO1 Salicided CMOS Process", Proceedings of 8th International Symposium on the Physical and Failure Analysis of Integrated Ciruits (IPFA), pp. 91 - 96, 9- 13 July, 2001, Singapore.

[6] Ming-Dou Ker and Che-Hao Chuang, "ESD Protection Circuits with Novel MOS-Bounded Diode Structures", IEEE Internation Symposium on Circuits and Systems (ISCAS), pp. V-533 V-536, vol.5, 26-29 May 2002, Arizona.

[7] M.-D. Ker, "Whole-Chip ESD Protection Design with Efficient VDD-to-VSS ESD Clamp Circuits for Submicron CMOS VLSI", IEEE Transactions on Electron Devices, Vol.46, No.1, January 1999.

[8] R. Merrill and E. Issaq, "ESD design methodology," EOS/ESD Symp. Proceedings, EOS-15, pp. 233-237, 1993.

[9] M.-D. Ker, C.-Y. Wu, H.-H. Chang, and T.-S. $\mathrm{Wu}$, "Whole-chip ESD protection scheme for CMOS mixed-mode IC's in deep-submicon CMOS technology," Proceedings of IEEE Custom Integrated Circuits Conference, pp. 31-34, 1997. 